06.3

\title{
Дважды отрицательные среды на основе антиферромагнитных полупроводников для терагерцевого диапазона частот
}

\author{
(C) С.В. Гришин ${ }^{1}$, А.В. Богомолова ${ }^{1}$, С.А. Никитов ${ }^{2}$ \\ ${ }^{1}$ Саратовский национальный исследовательский государственный университет им. Н.Г. Чернышевского, Саратов, Россия \\ ${ }^{2}$ Институт радиотехники и электроники им. В.А. Котельникова РАН, Москва, Россия \\ E-mail: sergrsh@yandex.ru
}

Поступило в Редакцию 9 июля 2021 г.

В окончательной редакции 16 декабря 2021 г.

Принято к публикации 16 декабря 2021 г.

\begin{abstract}
Приведены результаты теоретического исследования дисперсионных характеристик электромагнитных волн, существующих в поперечно намагниченном антиферромагнитном полупроводнике с потерями. Антиферромагнитный полупроводник представляет собой безграничную бигиротропную среду, эффективные материальные параметры которой являются дважды отрицательными в нескольких областях частот. Установлено, что данные частотные области находятся в терагерцевом диапазоне и в них существуют четыре обратные электромагнитные волны, две из которых являются ТЕ-волнами, а две другие - ТМ-волнами.
\end{abstract}

Ключевые слова: левые среды, антиферромагнетики, полупроводники, спиновые волны.

DOI: 10.21883/PJTF.2022.05.52156.18955

Дважды отрицательные среды (или „левые“ среды) являются одной из разновидностей метаматериалов, у которых диэлектрическая $(\varepsilon)$ и магнитная $(\mu)$ проницаемости являются одновременно отрицательными величинами [1]. Это приводит к появлению у среды отрицательного показателя преломления и распространению в ней обратной электромагнитной волны (ЭМВ). На данный момент дважды отрицательные среды созданы как для микроволнового, так и для терагерцевого $(\mathrm{THz})$ диапазона частот с использованием субволновых элементов (металлические тонкие проволоки и кольцевые разомкнутые резонаторы), размещенных, как правило, в немагнитной матрице [2].

В работе [3] было показано, что создать управляемую магнитным полем дважды отрицательную среду для THz-диапазона можно с использованием природного магнитного материала, которым является антиферромагнетик (АФМ). АФМ, как известно, представляет собой $\mu$-отрицательную среду, у которой из-за наличия двух подрешеток и сильного внутреннего магнитного поля магнитная проницаемость является отрицательной величиной в двух частотных областях, находящихся в THz-диапазоне [4]. Совмещение $\mu$-отрицательных свойств АФМ со свойствами $\varepsilon$-отрицательной среды, в качестве которой была выбрана периодическая решетка из тонких проводящих проволок, дало возможность реализовать дважды отрицательную среду без использования дополнительных субволновых элементов в виде кольцевых разомкнутых резонаторов.

Другим не менее интересным кандидатом для создания управляемой магнитным полем дважды отрицательной среды является бигиротропная среда [5]. Известно, что в бигиротропной среде связь векторов напряженности электрического и магнитного полей с векторами электрической и магнитной индукций осуществляется через материальные параметры среды (диэлектрическую и магнитную проницаемости), которые описываются эрмитовыми тензорами второго ранга [6]. Примером бигиротропного материала является магнитный полупроводник, который обладает анизотропными свойствами намагниченного магнетика ( $\mu$-отрицательная среда) и магнитоактивной электронной плазмы ( $\varepsilon$-отрицательная среда). В работе [1] впервые было обращено внимание на возможность создания дважды отрицательной среды из магнитного полупроводника. В [7] с использованием электродинамической модели поперечно намагниченного ферромагнитного полупроводника была показана трансформация дисперсионной характеристики (ДХ) медленной ЭМВ от положительной нормальной к отрицательной аномальной при изменении местоположения плазменной частоты относительно частот ферромагнитного резонанса и антирезонанса. Однако физического объяснения появления обратной ЭМВ здесь дано не было.

Целью настоящей работы является демонстрация возможности создания дважды отрицательной среды на

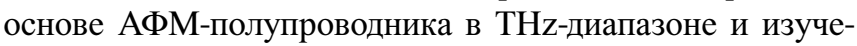
ния влияния потерь на дисперсионные характеристики обратных ЭМВ.

Рассмотрим бигиротропный материал, схематическое изображение которого приведено на рис. $1, a$. Он представляет собой намагниченный АФМ, в котором существуют свободные носители заряда в виде замагниченной „холодной“ плазмы. Внешнее постоянное магнитное поле $\mathbf{H}_{0}$ приложено вдоль оси 0Z. Для указанного материала, безграничного в направлении оси $0 X$, распространение ЭМВ происходит вдоль оси $0 Y$. Диэлектрические свойства 


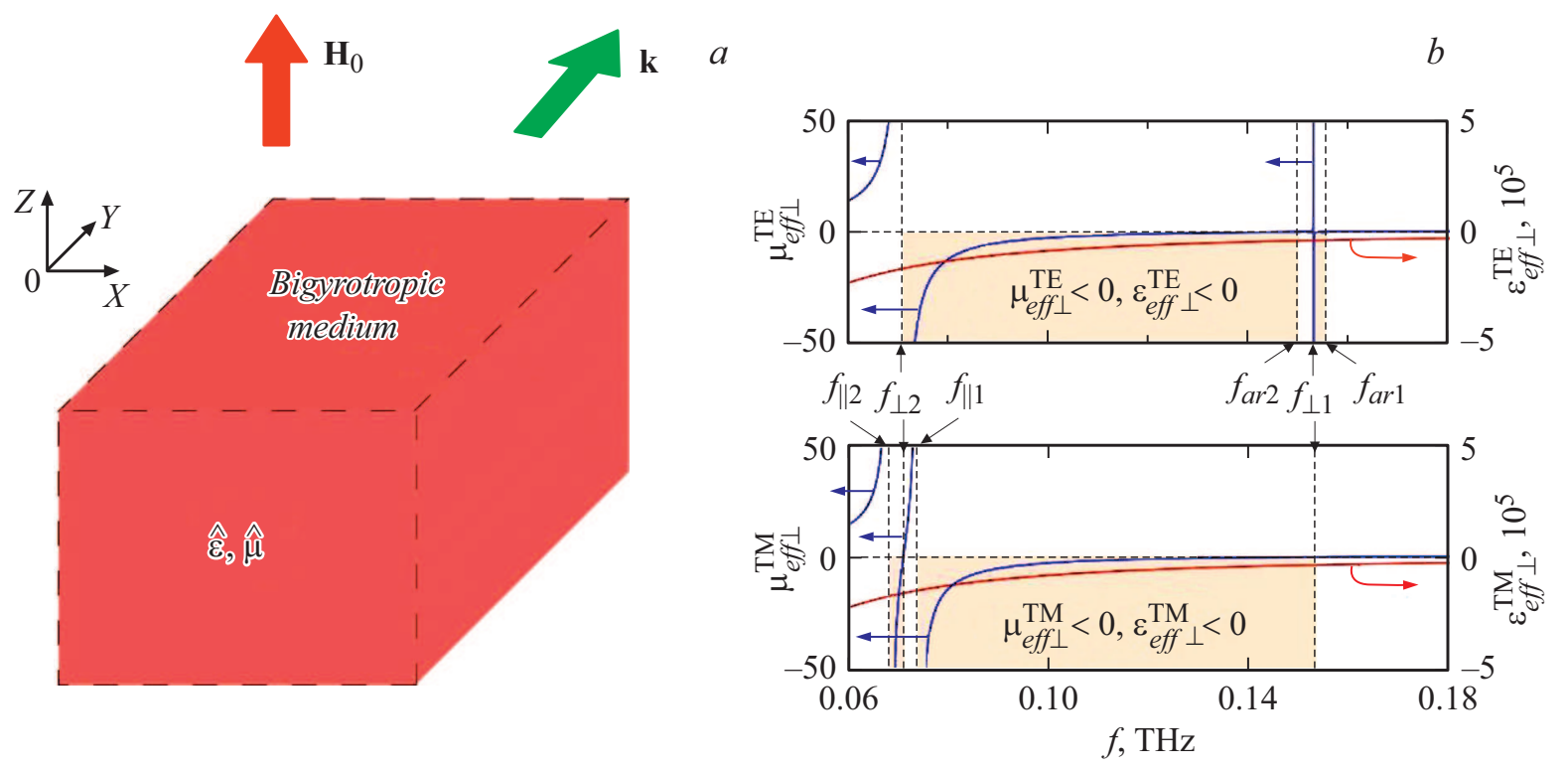

Рис. 1. $a-$ схематическое изображение поперечно намагниченной безграничной бигиротропной среды. $b-$ частотные зависимости эффективных высокочастотных электрических и магнитных проницаемостей поперечно намагниченной среды, в которой существуют ТЕ-волны (верхний фрагмент) и ТМ-волны (нижний фрагмент). Расчеты эффективных параметров среды выполнены для $H_{0}=79.58 \mathrm{kA} / \mathrm{m}, H_{E}=2.86 \mathrm{MA} / \mathrm{m}, H_{A}=636.64 \mathrm{kA} / \mathrm{m}, N=10^{19} \mathrm{~cm}^{-3}, M_{S}=1.16 \mathrm{~T}, \varepsilon_{r}=6.9$.

бигиротропного материала описываются тензором высокочастотной диэлектрической проницаемости, который с учетом потерь, обусловленных столкновением электронов, имеет следующий вид [5-8]:

$$
\overleftrightarrow{\varepsilon}=\varepsilon_{f}\left(\begin{array}{ccc}
\varepsilon & -j g & 0 \\
j g & \varepsilon & 0 \\
0 & 0 & \eta
\end{array}\right),
$$

$\varepsilon_{f}=\varepsilon_{0} \varepsilon_{r}-$ абсолютная диэлектрическая проницаемость среды, $\varepsilon_{0}=1 /\left(\mu_{0} c^{2}\right)$ - электрическая постоянная, $\mu_{0}-$ магнитная постоянная, $\varepsilon_{r}-$ относительная диэлектрическая проницаемость среды,

$$
\begin{gathered}
\varepsilon=1-\omega_{p e}^{2}\left(\omega-j v_{e}\right) /\left\{\omega\left[\left(\omega-j v_{e}\right)^{2}-\omega_{c e}^{2}\right]\right\}, \\
g=\omega_{p e}^{2} \omega_{c e} /\left\{\omega\left[\left(\omega-j v_{e}\right)^{2}-\omega_{c e}^{2}\right]\right\}, \\
\eta=1-\omega_{p e}^{2} / \omega\left(\omega-j v_{e}\right),
\end{gathered}
$$

$\omega_{p e}=\sqrt{4 \pi N e^{2} / m_{e}}-$ плазменная частота электронов, $\omega_{c e}=\left|e B_{0}\right| /\left(m_{e} c\right)$ - циклотронная частота электронов, $\omega=2 \pi f-$ круговая частота, $f-$ линейная частота, $v_{e}$ - частота столкновений электронов, $N-$ концентрация электронов в плазме, $e / m_{e}-$ удельный заряд электрона, $B_{0}$ - постоянная магнитная индукция. Магнитные свойства описываются тензором высокочастотной магнитной проницаемости в виде

$$
\overleftrightarrow{\mu}=\left(\begin{array}{ccc}
\mu & j \mu_{a} & 0 \\
-j \mu_{a} & \mu & 0 \\
0 & 0 & 1
\end{array}\right)
$$

где диагональные $\mu$ и недиагональные $\mu_{a}$ компоненты тензора для АФМ с „легкой“ осью анизотропии, совпадающей с осью $0 Z$, и потерями одинаковыми для двух подрешеток запишутся в следующем виде [3,4]:

$$
\begin{aligned}
\mu & =1+8 \pi \gamma_{S}^{2} M_{S} H_{A}\left[\left(\omega_{+}+j \alpha \omega\right)\left(\omega_{-}+j \alpha \omega\right)\right. \\
- & \left.\omega^{2}\right] /\left\{\left[\left(\omega_{+}+j \alpha \omega\right)^{2}-\omega^{2}\right]\left[\left(\omega_{-}+j \alpha \omega\right)^{2}-\omega^{2}\right]\right\}, \\
\mu_{a} & =8 \pi \gamma_{S}^{2} M_{S} H_{A} \omega\left(\omega_{-}-\omega_{+}\right) /\left\{\left[\left(\omega_{+}+j \alpha \omega\right)^{2}-\omega^{2}\right]\right. \\
& \left.\times\left[\left(\omega_{-}+j \alpha \omega\right)^{2}-\omega^{2}\right]\right\},
\end{aligned}
$$

где $\gamma_{S}$ - усредненный $g$-фактор, $M_{S}-$ усредненная статическая намагниченность подрешеток, $H_{A}$ - поле анизотропии, $\omega_{+}=\gamma_{S}\left(H_{C}+H_{0}\right), \omega_{-}=\gamma_{S}\left(H_{C}-H_{0}\right)-$ частоты антиферромагнитного резонанса, $H_{C}=\left[H_{A}\left(2 H_{E}+H_{A}\right)\right]^{1 / 2}-$ поле „опрокидывания“ подрешеток, $H_{E}-$ поле однородного обменного взаимодействия между подрешетками, $\alpha$ - параметр потерь, обусловленный процессами релаксации волн в АФМ.

При решении электродинамической задачи в приближении однородных плоских волн для поперечно намагниченной безграничной бигиротропной среды получаем два дисперсионных уравнения (ДУ), одно из которых описывает характеристики ТЕ-волн, а другое - ТМволн. Для ТЕ-волн ДУ имеет следующий вид:

$$
k=k_{0}\left(\mu_{e f f \perp}^{\mathrm{TE}} \varepsilon_{e f f \perp}^{\mathrm{TE}}\right)^{1 / 2},
$$

где $k$ - волновое число ЭМВ в среде, $k_{0}=\omega / c-$ волновое число ЭМВ в вакууме, $\mu_{e f f \perp}^{\mathrm{TE}}$ и $\varepsilon_{e f f \perp}^{\mathrm{TE}}-$ эффективные магнитная и диэлектрическая проницаемости, которые для среды с ТЕ-волнами определяются как

$$
\begin{gathered}
\mu_{e f f \perp}^{\mathrm{TE}}=\left(\mu^{2}-\mu_{a}^{2}\right) / \mu, \\
\varepsilon_{e f f \perp}^{\mathrm{TE}}=\varepsilon_{f} \eta .
\end{gathered}
$$



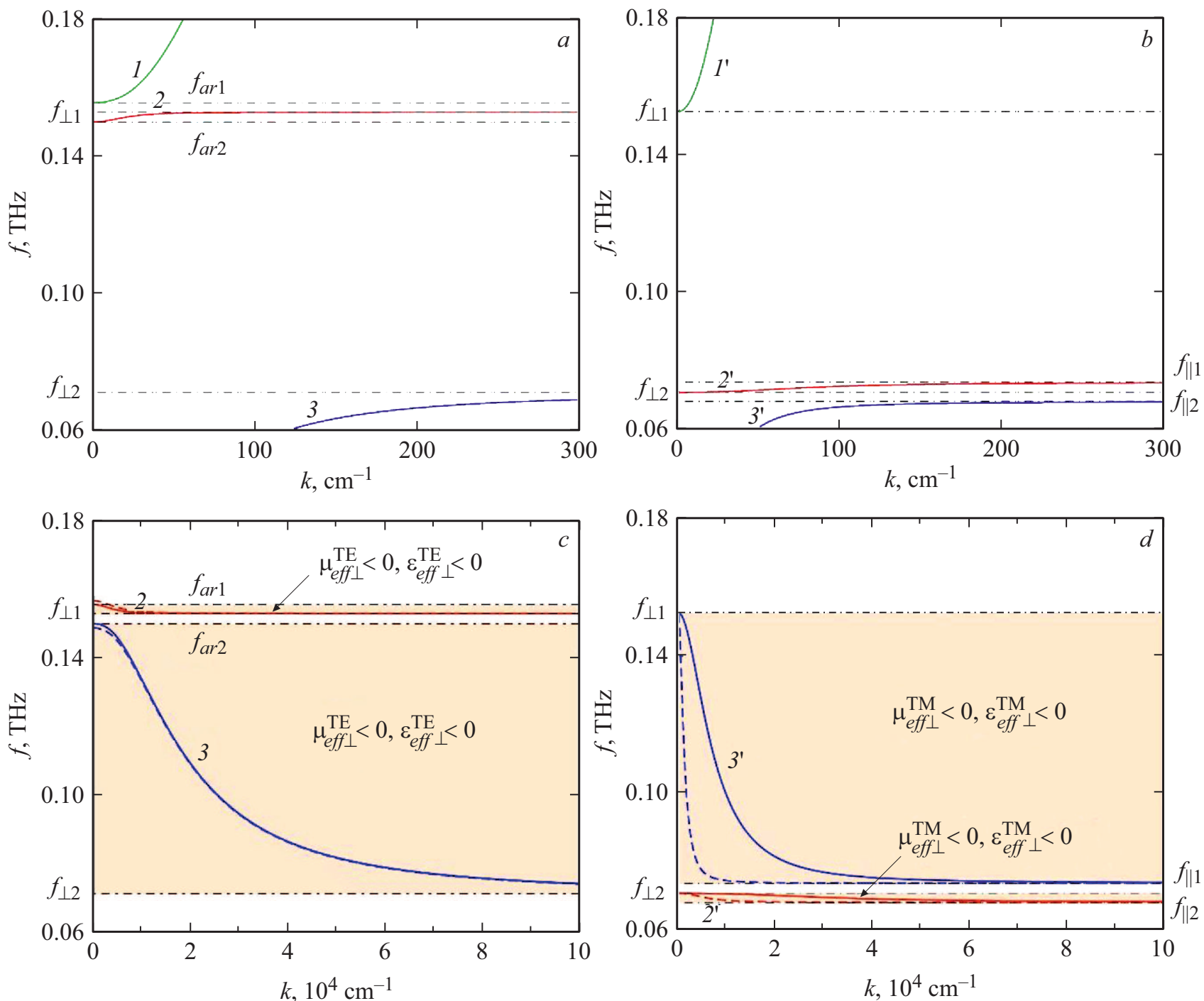

Рис. 2. Дисперсионные характеристики быстрых (сплошные линии $1,1^{\prime}$ ) и медленных (сплошные линии $2,2^{\prime}, 3,3^{\prime}$ и штриховые линии) ТЕ-волн $(a, c)$ и ТМ-волн $(b, d)$, существующих в поперечно намагниченном АФМ при $N=0$ и $\alpha=0(a, b)$ и АФМполупроводнике с $N=10^{19} \mathrm{~cm}^{-3}$ при $v_{e}=0, \alpha=0$ (сплошные линии) и $v_{e}=10^{14} \mathrm{rad} \cdot \mathrm{Hz}, \alpha=4 \cdot 10^{-2}$ (штриховые линии) $(c, d)$. На $(c)$ расчеты выполнены для $\alpha=4 \cdot 10^{-2}$ и $v_{e}=0$, а на $(d)-$ для $v_{e}=10^{14} \mathrm{rad} \cdot \mathrm{Hz}$ и $\alpha=0$. Расчеты выполнены для $H_{0}=79.58 \mathrm{kA} / \mathrm{m}, H_{E}=2.86 \mathrm{MA} / \mathrm{m}, H_{A}=636.64 \mathrm{kA} / \mathrm{m}, M_{S}=1.16 \mathrm{~T}, \varepsilon_{r}=6.9$.

Для ТМ-волн ДУ имеет следующий вид:

$$
k=k_{0}\left(\mu_{e f f \perp}^{\mathrm{TM}} \varepsilon_{e f f \perp}^{\mathrm{TM}}\right)^{1 / 2},
$$

где $\mu_{e f f \perp}^{\mathrm{TM}}$ и $\varepsilon_{e f f \perp}^{\mathrm{TM}}-$ эффективные магнитная и диэлектрическая проницаемости, которые для среды с ТМ-волнами равны

$$
\begin{gathered}
\mu_{e f f \perp}^{\mathrm{TM}}=\mu, \\
\varepsilon_{e f f \perp}^{\mathrm{TM}}=\varepsilon_{f}\left(\varepsilon^{2}-g^{2}\right) / \varepsilon .
\end{gathered}
$$

Условия, при которых $\mu_{e f f \perp}^{\mathrm{TE}}<0(\alpha=0)$, запишутся как

$$
\begin{aligned}
& \omega_{\perp 1}<\omega<\omega_{\text {ar } 1}, \\
& \omega_{\perp 2}<\omega<\omega_{\text {ar } 2},
\end{aligned}
$$

где

$$
\omega_{a r 1,2}=\left[ \pm\left(\omega_{+}-\omega_{-}\right)+D_{1}^{1 / 2}\right] / 2
$$

— частоты АФМ-антирезонанса,

$$
\omega_{\perp 1,2}=\left[\left(\omega_{+}^{2}+\omega_{-}^{2}+8 \pi \gamma_{S}^{2} M_{S} H_{A} \pm D_{2}^{1 / 2}\right) / 2\right]^{1 / 2}
$$

— частоты АФМ-резонанса при поперечном намагничивании,

$$
\begin{aligned}
D_{1} & =\left(\omega_{+}+\omega_{-}\right)^{2}+32 \pi \gamma_{S}^{2} M_{S} H_{A}, \\
D_{2} & =\left(\omega_{+}^{2}+\omega_{-}^{2}+8 \pi \gamma_{S}^{2} M_{S} H_{A}\right)^{2} \\
& -4 \omega_{+} \omega_{-}\left(\omega_{+} \omega_{-}+8 \pi \gamma_{S}^{2} M_{S} H_{A}\right) .
\end{aligned}
$$

Для существования двух обратных ТЕ-волн на частотах, определяемых из условий (9a) и (9b), 
необходимо, чтобы на данных частотах выполнялось $\varepsilon_{e f f \perp}^{\mathrm{TE}}<0\left(v_{e}=0\right)$. Такая ситуация возможна, когда

$$
\omega_{\perp 1,2}<\omega<\omega_{\text {ar } 1,2} \leqslant \omega_{p e} .
$$

Условия, при которых $\mu_{e f f \perp}^{\mathrm{TM}}<0(\alpha=0)$, запишутся как

$$
\begin{aligned}
& \omega_{\| 1}<\omega<\omega_{\perp 1}, \\
& \omega_{\| 2}<\omega<\omega_{\perp 2},
\end{aligned}
$$

где $\omega_{\| 1,2}=\omega_{ \pm}-$частоты АФМ-резонанса при продольном намагничивании.

Для существования двух обратных ТМ-волн на частотах, определяемых из условий (11a) и (11b), необходимо, чтобы на данных частотах выполнялось $\varepsilon_{e f f \perp}^{\mathrm{TM}}<0$ $\left(v_{e}=0\right)$. Такая ситуация возможна, например, когда

$$
\omega_{|| 1,2}<\omega<\omega_{\perp 1,2} \leqslant \omega_{\varepsilon},
$$

где $\omega_{\varepsilon}=\left[-\omega_{c e}+\left(\omega_{c e}^{2}+4 \omega_{p e}^{2}\right)^{1 / 2}\right] / 2$.

На рис. $1, b$ построены частотные зависимости эффективных параметров среды и заливкой выделены области частот, в которых могут существовать обратные ТЕ- и ТМ-волны. На рис. 2 приведены ДХ ТЕ- и ТМ-волн, которые рассчитаны на основе выражений (3) и (6) с учетом (4), (5), (7), (8) для АФМ-полупроводника, которому соответствует один из монохалькогенидов европия теллурид европия (EuTe) $[9,10]$. Здесь же для сравнения приведены ДХ ТЕ- и ТМ-волн, которые рассчитаны для АФМ, у которого отсутствуют свободные носители заряда $(N=0)$ и потери. Из представленных на рис. $2, a, b$ результатов расчета следует, что в АФМ существуют медленные ЭМВ только с нормальной дисперсией. Присутствие электронной плазмы в АФМ приводит к тому, что медленные ЭМВ становятся обратными волнами, которые существуют в THz-диапазоне частот, где эффективные параметры среды являются дважды отрицательными (рис. $2, c, d$ и $1, b$ ). Расчеты показали, что потери, обусловленные процессами спин-волновой релаксации, оказывают влияние в основном на ДХ ТЕволн, а потери в плазме влияют только на ДХ ТМ-волн. В первом случае замедление более низкочастотной обратной ТЕ-волны уменьшается, а более высокочастотной обратной ТЕ-волны, наоборот, увеличивается на фиксированной частоте. Что касается обратных ТМ-волн, то замедление у обеих (высокочастотной и низкочастотной) волн уменьшается с увеличением потерь в плазме.

В заключение отметим, что полученные результаты представляют интерес для разработки функциональных устройств $\mathrm{THz}-$ спинтроники.

\section{Финансирование работы}

Работа выполнена за счет гранта Российского научного фонда (проект № 19-79-20121).

\section{Конфликт интересов}

Авторы заявляют, что у них нет конфликта интересов.

\section{Список литературы}

[1] В.Г. Веселаго, УФН, $92 \quad$ (3), $517 \quad$ (1967). DOI: $10.3367 /$ UFNr.0092.196707d.0517 [V.G. Veselago, Sov. Phys. Usp., 10 (3), 509 (1968). DOI: 10.1070/PU1968v010n04ABEH003699].

[2] Metamaterials handbook. Phenomena and theory of metamaterials, ed. by F. Capolino (CRC Press, Taylor \& Francis Group, 2009), vol. I.

[3] С.В. Гришин, М.Д. Амельченко, Ю.П. Шараевский, C.А. Никитов, Письма в ЖТФ, 47 (18), 32 (2021). DOI: 10.21883/PJTF.2021.18.51470.18873

[4] A.Yu. Sharaevskaya, D.V. Kalyabin, E.N. Beginin, Y.K. Fetisov, S.A. Nikitov, J. Magn. Magn. Mater., 475, 778 (2019). DOI: 10.1016/j.jmmm.2018.11.130

[5] Э.Г. Локк, А.В. Луговской, С.В. Герус, Радиотехника и электроника, 66 (7), 662 (2021). DOI: $10.31857 / \mathrm{S} 003384942107007 \mathrm{X}$

[6] А.Г. Гуревич, Ферриты на сверхвысоких частотах (Физматлит, М., 1960).

[7] М. Стил, Б. Вюраль, Взаимодействие волн в плазме твердого тела, пер. с англ. (Атомиздат, М., 1973).

[8] Электродинамика плазмы, под ред. А.И. Ахиезера, 2-е изд. (Наука, М., 1974).

[9] А.С. Боровик-Романов, С.О. Демокритов, Н.М. Крейнес, В.И. Кудинов, ЖЭТФ, 88 (4), 1348 (1985). [A.S. BorovikRomanov, S.O. Demokritov, N.M. Kreines, V.I. Kudinov, Sov. Phys. JETP, 61 (4), 801 (1985).].

[10] В.В. Павлов, ФТТ, $61 \quad$ (3), $539 \quad$ (2019). DOI: 10.21883 /FTT.2019.03.47248.263 [V.V. Pavlov, Phys. Solid State, 61 (3), 408 (2019). DOI: $10.1134 / \mathrm{S} 1063783419030247]$. 